\title{
X-ray Fluorescent Determination of Lanthanum, Cerium, Praseodymium, and Neodymium in Plain Carbon and Low Alloy Steels*
}

\author{
By Kïchi NARITA, ${ }^{* *}$ Tetsuo MATSUMURA, ${ }^{* *}$ Renpei MOROOKA, ${ }^{* *}$ \\ Naomi KOTANI,** and Takeshi GOTO**
}

\begin{abstract}
Synopsis
An investigation was undertaken to determine four rare earth elements ( $\mathrm{La}, \mathrm{Ce}, \mathrm{Nd}$, and $\mathrm{Pr}$ ) in the range of 0.005 to $0.15 \%$ by the $X$-ray fluorescent spectrometry.

In the absence of reliable standards, the glass bead technique for irregularly shaped samples such as drillings was developed. Rare earth elements were precipitated as the fluorides after dissolving $5 \mathrm{~g}$ of the sample in sulfuric acid, the filtered fluorides were then ignited to oxides, mixed with sodium tetraborate, and melted to prepare the glass beads.

The glass bead method ensured a precision of less than $2 \%$ of the amount present. A good agreement was obtained between the chemical and X-ray results.

The advantages of the method were the facility of the construction of an analytical curve by using chemical reagents, the absence of interelemental effects because of the separation of rare earth elements, and the versatility to be applicable to other materials with minor modifications.

The standard samples of low alloy steels for the direct metal disk technique were furthermore prepared and a conventional procedure was taken with satisfactory results.
\end{abstract}

\section{Introduction}

Recent metallurgical research shows that the addition of cerium and other rare earth metals improves the mechanical properties of steel. But, the effect of rare earth elements on steel is complicated and its mechanism is not known in detail. Thus, a need arose for a reliable analytical method of individual rare earth elements in steel. Since the chemical properties of rare earth elements are very similar, their separation is quite difficult, and usually they are determined as total rare earths in chemical methods.

An X-ray fluorescent technique has been applied by many workers to the analysis of rare earth elements in various materials because of simplicity of spectra, rapidity, and other advantages. Only a few works, however, have been reported on steel samples. A direct X-ray fluorescent technique ${ }^{1)}$ is limited by lack of suitable solid standards. Kashuba and Hines ${ }^{2)}$ reported an ion exchange-pellet method, but neodymium could not be determined because of serious interference from chromium.
The present paper describes the development of an accurate method for the determination of lanthanum, cerium, praseodymium, and neodymium in plain carbon and low alloy steels. The method is based upon precipitation and recovery of the rare earths as fluorides from the sample solution, with subsequent ignition to oxides, fusion with sodium borate, and $\mathrm{X}$ ray fluorescent determination.

A direct metal disk technique has also been attempted and was found to be suitable for routine analysis because of its rapidity.

\section{Experimental}

\section{Apparatus and Reagents}

The equipment used is a Hilger and Watts Fluroprint $\mathrm{Mk}-\mathrm{II}$ with Philips tungsten target tube. Bunzen burners, platinum $-5 \%$ gold crucibles, graphite molds, etc. were used in the fusion technique. Anhydrous sodium tetraborate $\left(\mathrm{Na}_{2} \mathrm{~B}_{4} \mathrm{O}_{7}\right)$ was used as flux. Synthetic standards were prepared using high-purity oxides of the rare earth elements $(99.9 \%$ or over). Standard solutions containing $1 \mathrm{mg}$ of the rare earths per $\mathrm{m} l$ were also prepared by dissolving the oxides in sulfuric acid $(1+1)$, adding hydrogen peroxide if necessary and diluting as required.

\section{Preparation of Standard Steel Samples for Metal Disk Technique}

Since standard steel samples with suitable rare earths concentrations were not commercially available, a series of standard samples, six samples for each of the rare earth elements, were prepared by melting electrolytic iron, pure rare earth metals having a purity of better than $99.5 \%$, and other alloying metals in a vacuum induction furnace. The ingots obtained were forged to $40 \mathrm{~mm}$ dia. bars, and then the bars were homogenized at $1150^{\circ} \mathrm{C}$ for $20 \mathrm{hr}$. Disk samples (30 mm dia.) were machined from the bars. As an example, chemical compositions of the standards containing lanthanum are shown in Table 1.

Table 1. Chemical compositions of La standard samples (\%)

\begin{tabular}{|c|c|c|c|c|c|c|c|c|c|c|}
\hline Sample & C & $\mathrm{Si}$ & $\mathrm{Mn}$ & $\mathrm{P}$ & $\mathrm{S}$ & $\mathrm{Cu}$ & $\mathrm{Ni}$ & $\mathrm{Cr}$ & Mo & $\mathrm{La}$ \\
\hline $\mathrm{La}-1$ & 0.37 & 0.10 & 0.62 & 0.007 & 0.005 & 0.02 & 0.63 & 1.17 & 0.31 & 0.010 \\
\hline 2 & 0.40 & 0.15 & 0.59 & 0.006 & 0.004 & 0.02 & 0.40 & 1.60 & 0.10 & 0.011 \\
\hline 3 & 0.40 & 0.18 & 0.71 & 0.006 & 0.005 & 0.02 & 1.23 & 0.63 & 0.06 & 0.047 \\
\hline 4 & 0.40 & 0.17 & 0.68 & 0.005 & 0.005 & 0.02 & 0.79 & 0.30 & 0.14 & 0.106 \\
\hline 5 & 0.39 & 0.25 & 0.61 & 0.006 & 0.004 & 0.02 & 0.97 & 0.75 & 0.21 & 0.156 \\
\hline
\end{tabular}

* Originally published in Tetsu-to-Hagané, 59 (1973), 1159, in Japanese. English version received November $15,1973$.

** Central Research Laboratory, Kobe Steel Ltd., Wakinohama-cho, Fukiai-ku, Kobe 651. 


\section{Instrumental Conditions}

In X-ray analysis of rare earth elements the use of $K$ lines has considerable advantages over that of $L$ lines. ${ }^{3,4)}$ However, in this investigation characteristic X-ray lines of the $L$ series were used because of the instrumental limitations such as operating voltage $(50 \mathrm{kV}$ maximum) of the $\mathrm{X}$-ray tube. Lines that may coincide or overlap when exciting $L$ lines are $\mathrm{Ce}$ $L_{\beta_{1}}$ for $\mathrm{Nd} L_{\alpha_{1}}$, La $L_{\beta_{1}}$, and V $K_{\alpha_{1}}$ for Pr $L_{\alpha_{1}}$, and Cr $K_{\alpha_{1}}$ for $\operatorname{Pr} L_{\beta_{1}}$. For the determination of praseodymium the $\operatorname{Pr} L_{\alpha_{1}}$ was preferred to the $\operatorname{Pr} L_{\beta_{1}}$ because of the high chromium content in the alloy steels of interest. Therefore, the praseodymium measurements were corrected for the interference from lanthanum and vanadium. Analytical lines used for other elements are Nd $L_{\beta_{1}}$, Ce $L_{\alpha_{1}}$, and La $L_{\alpha_{1}}$, which are free from interfering lines. Other instrumental conditions are summarized in Table 2.

\section{Results and Discussion}

1. Glass Bead Technique

\section{Preparation of Glass Bead Samples}

The rare earth elements were separated from the sample solution as fluorides by a wet chemical method, ${ }^{5)}$ ignited to oxides, and fused into a $30 \mathrm{~mm}$ dia. glass bead. Although 10 to $15 \mathrm{~g}$ of flux is usually used

Table 2. Operating conditions

\begin{tabular}{l|l} 
X-ray tube & W-target, $50 \mathrm{kV}-20 \mathrm{~mA}$ \\
Collimator & $1 \mathrm{st} 0.01 \times 4 \mathrm{in}, 2 \mathrm{nd} 0.025 \times 1 \mathrm{in}$ \\
Crystal & Flat LiF \\
Detector & $\mathrm{SC}+\mathrm{PC}$ \\
P. H. A. & $12.5 \mathrm{~V}$ Disc. \\
Mode & Monitor system, $105 \mathrm{sec}$
\end{tabular}

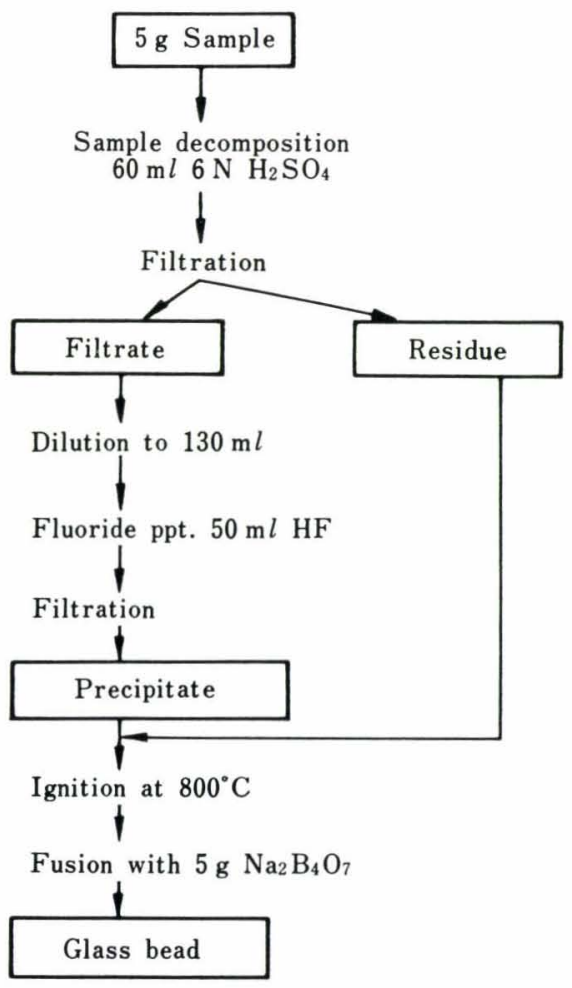

Fig. 1. Schematic procedure for sample preparation in the fusion, ${ }^{6}$ ) in this work, in view of the sensitivity of the method and other considerations, the rare earth oxides were fused with only $5 \mathrm{~g}$ of sodium tetraborate.

As shown schematically in Fig. 1, the sample is dissolved in dilute sulfuric acid, and the rare earth elements precipitated as fluorides. The fluorides together with the acid insoluble residue are transferred to a platinum $-5 \%$ gold crucible and ignited to the oxides. The oxides are mixed with $5 \mathrm{~g}$ of the flux and heated at about $1000^{\circ} \mathrm{C}$ for 5 to $10 \mathrm{~min}$. When completely fused the content is thoroughly homogenized by swirling by the crucible and is poured into a graphite mold previously heated at $350^{\circ}$ to $400^{\circ} \mathrm{C}$ on a hot plate. The melt spreads evenly when assisted by a graphite rod and is cooled slowly. X-ray measurements are made on the bottom side of the bead thus prepared.

\section{Preparation of Synthetic Standard Samples}

Of the two ways of preparing standard beads for the fusion method, one from the samples of known concentration $^{6)}$ and the other from the pure (spectroscopic grade) chemical reagents, ${ }^{7)}$ the latter was adopted in this investigation because such synthetic standards can be used without running check analysis. Table 3 shows the prepared standards containing 0.005 to $0.2 \%$ of each of the rare earth elements. Another set of standards containing one element was also prepared. These standards were made by adding standard rare earth solutions to a rare earth free base material (a $0.3 \%$ Ni-0.2\% Cr $-0.1 \%$ Mo-0.2\% V steel) and by following the procedure shown in Fig. 1. In addition, for the purpose of comparison the standard beads covering the same concentration range were fused by direct fusion of the rare earth oxides, as much as $15 \mathrm{~g}$ of sodium borate being used in this case.

\section{Effect of Sample Thickness on Background Intensity}

As an example of the analytical curves prepared from the standard beads described above, the curves

Table 3. Synthetic standard samples (\%)

\begin{tabular}{c|c|c|c|c}
\hline Sample & Nd & Pr & Ce & La \\
\hline S-1 & 0.200 & 0.005 & 0.200 & 0.005 \\
2 & 0.100 & 0.010 & 0.100 & 0.010 \\
3 & 0.050 & 0.020 & 0.050 & 0.020 \\
4 & 0.020 & 0.050 & 0.020 & 0.050 \\
5 & 0.010 & 0.100 & 0.010 & 0.100 \\
6 & 0.005 & 0.200 & 0.005 & 0.200
\end{tabular}

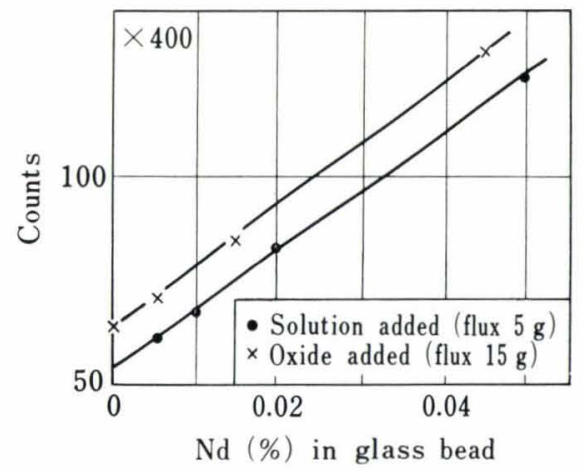

Fig. 2. Comparison of glass bead preparation techniques 
for neodymium are shown in Fig. 2. Distinct difference or bias was found between the two curves which were based on two sets of standards prepared by solution addition and oxide fusion methods. Incomplete recovery of the element in chemical separation, contamination of interfering elements, and the difference in the amount of flux were considered to be possible reasons for that, and therefore an investigation was carried out on them.

As the result, it was found that the background intensity varied with sample thickness. In Fig. 3, are shown the results of X-ray measurments made on blank beads of varying thickness at the diffraction angle of neodymium. Glass beads fused with 15 and $5 \mathrm{~g}$ of sodium borate are about 8.5 and $2.5 \mathrm{~mm}$ thick, respectively, and a $5 \mathrm{~g}$ borax bead is not thick enough to attain critical thickness as can be seen from the figure.

When corrections were made for sample thickness according to the relation curve given in Fig. 3, the two analytical curves in Fig. 2 were found to become identical within experimental error. Therefore, the discrepancy in the analytical curves in question is considered to have been caused by continuous X-ray radiation whose intensity varies with sample thickness. However, the effect of sample thickness gives no practical difficulties when the same amount of borax is used for the preparation of both standards and unknowns.

The agreement of the two analytical curves explained above shows also that the rare earths can be recovered quantitatively by the fluoride precipitation method because the standards made by the solution addition method have been prepared following all the steps of the procedure shown in Fig. 1.

\section{Effect of Extraneous Elements from Acid Insoluble Residue}

After the steel samples had been treated according to the procedure shown in Fig. 1, the acid insoluble residues were fused and contained in glass beads. Fiure 4 shows the X-ray spectrogram of a glass bead obtained from a Ni-Cr-Mo-V steel sample. Obviously, the acid insoluble residue contains considerable amounts of iron, nickel, copper, etc. Upon examination of the mass absorption coefficients of these elements for the rare earth elements, there found little difference among them. Therefore, as a typical example the effect of iron on praseodymium determinations was studied.

About $10 \mathrm{mg}$ of iron was found in the residue by preliminary chemical analysis; therefore glass beads containing iron in amounts up to $20 \mathrm{mg}$ or $0.4 \%$ based on a $5 \mathrm{~g}$ sample were prepared. Iron was added as solution to the fluoride obtained by treating a low alloy steel sample containing $0.058 \%$ of praseodymium, and the procedure in Fig. 1 was followed with the exception that the insoluble residue was discarded. As presented in Table 4, the analytical results are in close agreement regardless of the presence of iron. There fore, it can be said that the extraneous elements encountered in the residue will not give any effect on the $\mathrm{X}$-ray determination of rare earth elements so far as absorbing and enhancing effects are concerned.

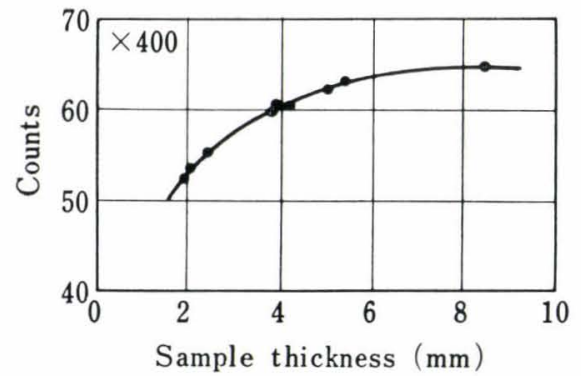

Fig. 3. Relation between sample thickness and X-ray intensity $\left(\mathrm{Nd} L_{\beta_{1}}\right)$

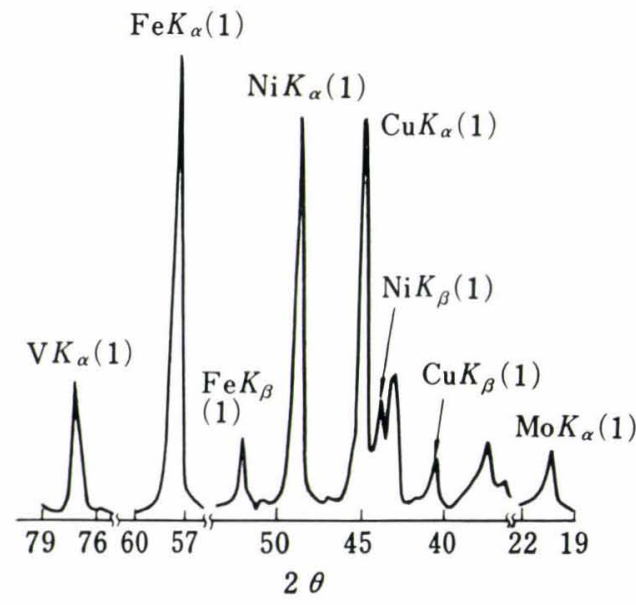

Fig. 4. Qualitative analysis of acid insoluble residue of steel $(\mathrm{Fe}-0.3 \mathrm{Ni}-0.2 \mathrm{Cr}-0.1 \mathrm{M} \circ-0.2 \mathrm{~V})$

Table 4. Effect of iron on X-ray analytical results of $\mathrm{Pr}$

\begin{tabular}{c|c|c}
\hline Sample & Fe added $(\%)$ & $\operatorname{Pr}(\%)$ \\
\hline F-1 & 0.00 & 0.058 \\
2 & 0.05 & 0.058 \\
3 & 0.10 & 0.059 \\
4 & 0.20 & 0.058 \\
5 & 0.30 & 0.056 \\
6 & 0.40 & 0.058 \\
\hline
\end{tabular}

Table 5. Effect of treatment of acid insoluble residue on $\mathrm{Pr}$ and La determinations

\begin{tabular}{c|cc}
\hline Sample & Pr or La $(\%)$ \\
\hline Pr-1 & Discarded & Combined \\
\hline Pr-2 & 0.004 & 0.007 \\
Pr-3 & 0.008 & 0.008 \\
Pr-4 & 0.021 & 0.021 \\
Pr-5 & 0.041 & 0.041 \\
Pr-6 & 0.058 & 0.056 \\
\hline La-2 & 0.059 & 0.059 \\
La-6 & 0.044 & 0.043 \\
\hline
\end{tabular}

Further investigation was carried out as to whether rejection of the insoluble residue would result in loss of the rare earths or not. Table 5 shows that the presence of the residue in glass beads has no relation with the analytical results. Therefore, it is concluded that 


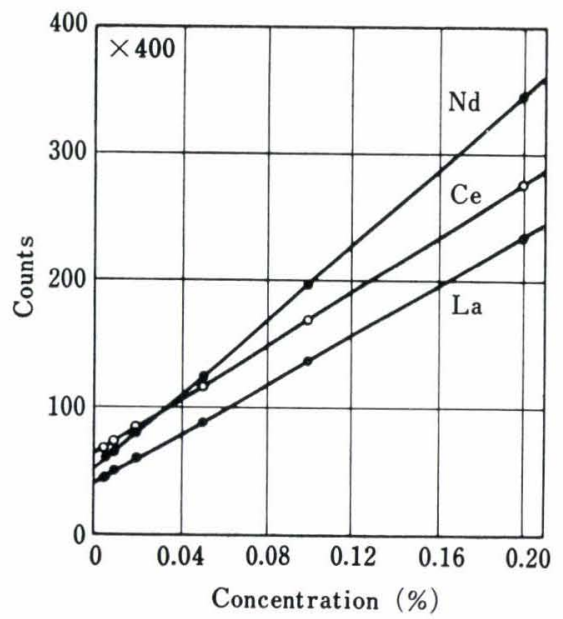

Fig. 5. Analytical curves for $\mathrm{La}, \mathrm{Ce}$, and $\mathrm{Nd}$ (Glass bead)

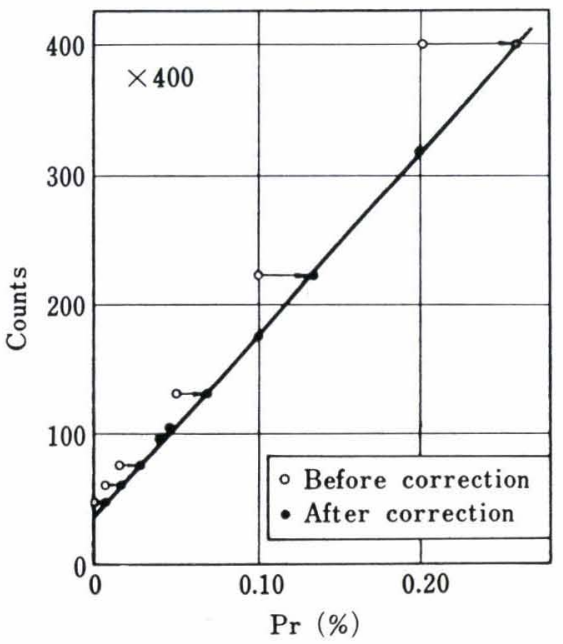

Fig. 6. Analytical surve for $\operatorname{Pr}$ (Glass bead)

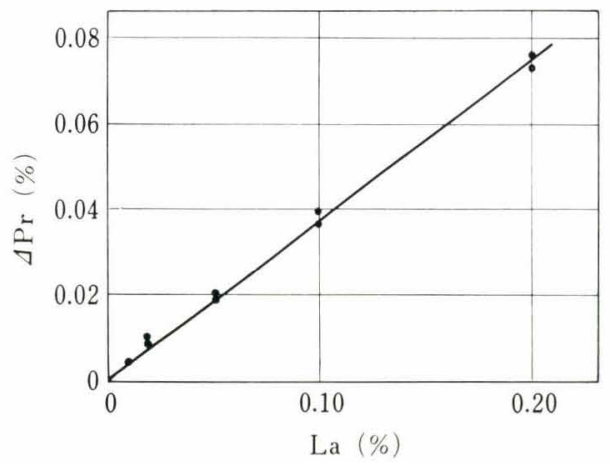

Fig. 7. Correction for La in $\mathrm{Pr}$ determination

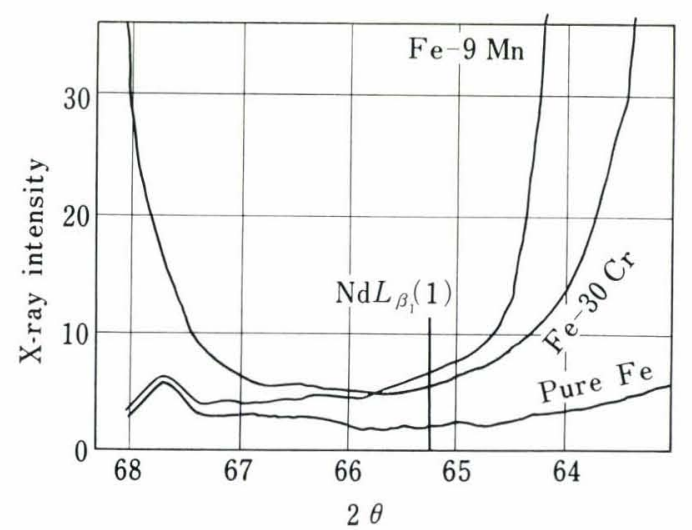

Fig. 8. Interferences of $\mathrm{Mn}$ and $\mathrm{Cr}$ in $\mathrm{Nd} L_{\beta_{1}}(1)$ measurement (Metal disk) the rare earth elements are not present in appreciable amounts in the acid insoluble residue and also that such elements as nickel, copper, and molybdenum introduced from the residue do not affect the present method. The rejection of the insoluble residue not only simplifies the sample preparation procedure but also eliminates a correction procedure for vanadium which will be stated below. However, it will be advisable to ensure that steel samples to be analyzed do not contain any acid insoluble rare earths compounds.

\section{Analytical Curves}

Figures 5 and 6 show the analytical curves prepared from the synthetic standard glass beads. Satisfactory analytical curves were obtained for lanthanum, cerium, and neodymium. In the measurements of praseodymium there found considerable interference from lanthanum and vanadium due to the overlap of their characteristic X-ray lines. In order to study the interference from lanthanum, synthetic standard glass beads containing known amounts of the element were analyzed at the diffraction angle of $\operatorname{Pr} L_{\alpha_{1}}$ line. The $\mathrm{X}$-ray intensities were converted to the corresponding concentration of praseodymium, designated as $\Delta \mathrm{Pr}$, by refering to an analytical curve for praseodymium. Figure 7 shows the relation curve between $\triangle \operatorname{Pr}$ and lanthanum contents, $1 \%$ of lanthanum being equivalent to $0.38 \%$ of praseodymium.

A similar correction was made for vanadium. Vanadium, which will probably be present as nitride and carbide in the insolubele residue, interfers with the determination of praseodymium because the $\mathrm{V} K$ line cannot be resolved completely from the $\operatorname{Pr} L_{\alpha_{1}}$ line. From the relation curve of $\Delta \mathrm{Pr}$ and vanadium content, the percentage of praseodymium corresponding to $1 \%$ of vanadium was calculated as $0.022 \%$. When these corrections were made, the results of X-ray determinations on the synthetic standard glass beads containing lanthanum and vanadium were plotted with satisfactory accuracy on the analytical curve based on the synthetic standards containing praseodymium alone.

\section{Direct Metal Disk Technique}

In metal disk method, the sensitivity may be inferior to the glass bead method with chemical pretreatment for determination of trace elements, and a comprehensive library of standards closely resembling the unknowns in compositions may be necessary if results of the highest reliability are required. However, it offers advantages in respect to convenience and speed over the glass bead method, which is a reason why the direct metal disk method was investigated.

The sample surface was finished with \#120 alumina abrasive paper. The analytical problem then faced in the investigation of the method was only the correction for overlapping by neighboring lines such as La $L_{\beta_{1}}$ for $\operatorname{Pr} L_{\alpha_{1}}$ and tailing of $\mathrm{Cr} K_{\beta}$ and $\mathrm{Mn} K_{\alpha}$ for $\mathrm{Nd}$ $L_{\beta_{1}}$ which was not found in the glass bead technique. Figure 8 shows interferences of chromium and manganese in $\mathrm{Nd} L_{\beta_{1}}$ measurement, The degree of this 
effect depends on the variation of the background intensity due to matrix which results in the errors in the determination of neodymium.

\section{Detectability}

In Table 6, the detectabilities of the two analytical methods, as calculated from the equation defined by H. Neff, ${ }^{8)}$ are shown. The detectable concentrations are the least for praseodymium and the greatest for cerium in the glass bead method and for neodymium in the metal disk method. The higher background levels of $\mathrm{Ce} L_{\alpha_{1}}$ and $\mathrm{Nd} L_{\beta_{1}}$, because of overlapping of $\mathrm{W} L_{\beta_{1}}$ (2) from target material and tailings of Cr and $\mathrm{Mn} K$ lines, respectively, results in the lower detectabilities.

For samples of the critical thickness and above, the critical depth which is determined by the density and by mass absorption coefficient of the sample for the incident beam, would contribute to the detectability. Characteristic X-rays from the specimen of light element matrix $\left(\mathrm{Na}_{2} \mathrm{~B}_{4} \mathrm{O}_{7}\right)$ are radiated more efficiently than those from one of heavy matrices (iron).

\section{Precision and Accuracy}

The precision attained in the work can be assessed from Tables 7 and 8 . Table 7 shows the data on the repeatability calculated from 10 replicate determinations of a single glass bead or metal disk sample. It will be seen that the repeatability indicates the glass bead technique to be more precise than the metal disk technique. Table 8 shows the reproducibility of the glass bead technique obtained by carrying separate samples through the entire procedure. The reproducibility was less than $2 \%$ coefficient of variation and was at almost the same level as the repeatability was.

As an example of the data on the accuracy, the quantitative results of lanthanum analysis by the glass bead method are compared with those by the chemical analysis conducted by the "neo-thoron " spectrophotometry ${ }^{9)}$ in Table 9 . It will be seen in Table 9 that

Table 6. Detectability of methods (\%)

\begin{tabular}{c|cc}
\hline Element & Glass bead & Metal disk \\
\hline Nd & 0.00094 & 0.00496 \\
Pr & 0.00075 & 0.00180 \\
$\mathrm{Ce}$ & 0.00154 & 0.00255 \\
$\mathrm{La}$ & 0.00119 & 0.00219
\end{tabular}

$C_{d}=u(\alpha) \sqrt{2} \mathcal{N}_{b} \cdot C_{s} /\left(\mathcal{N}_{s}-\mathcal{N}_{b}\right)^{8)}$

$C_{d}$ : Detectability, $u(\alpha)$ : significance level, $\mathcal{N}_{b}$ : Counts of background, $\mathcal{N}_{s}$ : Counts of tested sample, $C_{s}$ : Concentration of tested sample
Table 8. Reproducibility of glass bead technique

\begin{tabular}{l|ll}
\hline$n$ & Pr $(\%)$ & Ce $(\%)$ \\
\hline 1 & 0.058 & 0.186 \\
2 & 0.059 & 0.184 \\
3 & 0.058 & 0.185 \\
4 & 0.056 & 0.184 \\
5 & 0.058 & 0.186 \\
6 & 0.058 & 0.184 \\
7 & - & 0.184 \\
8 & -0.184 \\
\hline$x$ & 0.00098 & 0.1846 \\
$\sigma$ & 1.695 & 0.00092 \\
$\mathrm{CV}$ & & 0.498 \\
\hline
\end{tabular}

Table 9. Comparison of chemical and X-ray results (\%)

\begin{tabular}{r|ccc} 
Sample & X-ray (Glass bead) & Chem. & $d$ \\
\hline La-1 & 0.008 & 0.010 & -0.002 \\
2 & 0.044 & 0.047 & -0.003 \\
3 & 0.011 & 0.011 & 0 \\
4 & 0.106 & 0.106 & 0 \\
5 & 0.150 & 0.156 & -0.006 \\
6 & 0.182 & 0.188 & -0.006 \\
$d$ & & & -0.0028 \\
$\sigma_{d}$ & & & 0.00271
\end{tabular}

Table 10. Analytical results of rare earth elements in steels $(\%)$

\begin{tabular}{|c|c|c|c|c|}
\hline \multirow{2}{*}{ Sample } & \multirow{2}{*}{ Element } & \multirow{2}{*}{ Chem. } & \multicolumn{2}{|c|}{ X-ray } \\
\hline & & & Glass bead & Metal disk \\
\hline \multirow{5}{*}{$A$} & La & - & 0.049 & 0.054 \\
\hline & $\mathrm{Ce}$ & - & 0.012 & 0.011 \\
\hline & $\operatorname{Pr}$ & - & 0.001 & 0.001 \\
\hline & $\mathrm{Nd}$ & 一 & 0.007 & 0.008 \\
\hline & T. RE & 0.074 & 0.069 & 0.074 \\
\hline \multirow{5}{*}{$B$} & $\mathrm{La}$ & - & 0.013 & 0.015 \\
\hline & $\mathrm{Ce}$ & - & 0.046 & 0.046 \\
\hline & $\operatorname{Pr}$ & - & 0.006 & 0.006 \\
\hline & $\mathrm{Nd}$ & - & 0.020 & 0.025 \\
\hline & T. RE & 0.082 & 0.085 & 0.092 \\
\hline \multirow{5}{*}{$C$} & $\mathrm{La}$ & - & 0.031 & 0.032 \\
\hline & $\mathrm{Ce}$ & - & 0.092 & 0.096 \\
\hline & $\operatorname{Pr}$ & - & 0.010 & 0.010 \\
\hline & Nd & - & 0.039 & 0.045 \\
\hline & T. RE & 0.169 & 0.172 & 0.183 \\
\hline
\end{tabular}

Table 7. Repeatability $(n=10) \quad(\%)$

\begin{tabular}{|c|c|c|c|c|c|c|}
\hline \multirow{2}{*}{ Element } & \multicolumn{3}{|c|}{ Glass bead } & \multicolumn{3}{|c|}{ Metal disk } \\
\hline & $\bar{x}$ & $\sigma$ & $\mathrm{CV}$ & $\bar{x}$ & $\sigma$ & $\mathrm{CV}$ \\
\hline $\operatorname{Pr}$ & 0.0582 & 0.00042 & 0.724 & 0.0575 & 0.00097 & 1.686 \\
\hline $\mathrm{Ce}$ & 0.1832 & 0.00092 & 0.502 & 0.1883 & 0.00082 & 0.435 \\
\hline $\mathrm{La}$ & 0.1487 & 0.00067 & 0.450 & 0.1480 & 0.00094 & 0.635 \\
\hline
\end{tabular}


the agreement is very satisfactory considering the uncertainties in the results of chemical analyses.

The typical analytical results of the routine samples are presented in Table 10. Sample $A$ was treated with lanthanum rich misch metal, and $B$ and $C$ with cerium rich misch metal. For the chemical method, which is incapable to separate each element, the results are shown as the total rare earths, while for the Xray method individual element is given. The agreement between those two methods is again quite satisfactory. Therefore, it can be said that the determination of the four principal rare earth elements studied here can give sufficient information on the rare earths content in steels treated with commercial rare earths additives such as misch metal and its alloys.

\section{Conclusion}

Chemical determination of rare earth elements in steels can be very laborious and costly, especially for the routine analysis. A fluorescent X-ray analysis method combining chemical treatment and glass bead sample technique has been developed in order to overcome these difficulties. Not only this method affords versatility and rapidity in analysis, but takes full advantage of the easiness in synthesizing the standard samples, in avoiding the interelement effects by se- parating elements from heavy matrix and diluting in light matrix, and in handling irregularly shaped samples.

Although these advantages are not obtainable in the fluorescent X-ray analysis using direct metal disk technique, for which many standard samples must be prepared in accordance with the concentration ranges of the steel samples of interest, its speed and convenience are valuable assets for routine works.

\section{REFERENCES}

1) I. Kawashima, T. Miyazaki, I. Tanaka, and K. Tokiwa: J. Spectroscopical Soc. Japan, 16 (1967), 14.

2) A. T. Kashuba and C. R. Hines: Anal. Chem., 43 (1971), 1758.

3) Y. Takahashi and E. Asada: Japan Analyst, 11 (1962), 926.

4) N. Matano: Japan Analyst, 21 (1972), 1187.

5) Japan Soc. for the Promotion of Science, 19th Committee (eds.): Tekko Kagaku Bunseki Zensho, No. 7, (1963), 162, Nikkan Kogyo Press.

6) T. Mizuno, T. Matsumura, N. Kotani, and T. Goto: J. Japan Inst. Metals, 31 (1967), 1186.

7) T. Mizuno, T. Matsumura, N. Kotani, and T. Goto: J. Japan Inst. Metals, 34 (1970), 476.

8) H. Neff: Arch. Eisenhüttenw., 34 (1963), 903.

9) S. Shono: Report of Japan Soc. for the Promotion of Science, 19th Committee, (1971), 19-9302. 